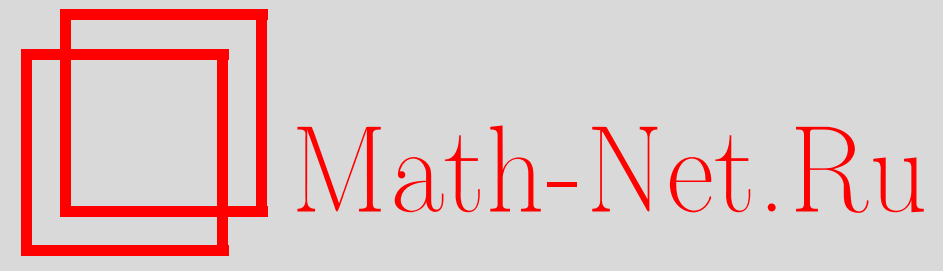

Б. П. Харламов, Финальное распределение диффузионного процесса: полумарковский подход, Теория вероятн. и ее примен., 2015, том 60, выпуск 3, 506-524

DOI: https://doi.org/10.4213/tvp4635

Использование Общероссийского математического портала Math-Net.Ru подразумевает, что вы прочитали и согласны с пользовательским соглашением http://www.mathnet.ru/rus/agreement

Параметры загрузки:

IP: 3.85 .7 .115

26 апреля 2023 г., 18:37:00

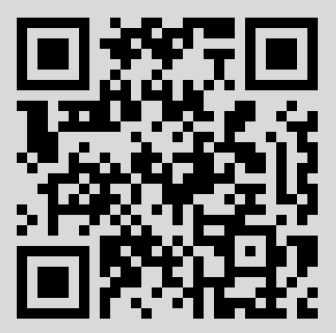




\title{
ФИНАЛЬНОЕ РАСПРЕДЕЛЕНИЕ ДИФФУЗИОННОГО ПРОЦЕССА: ПОЛУМАРКОВСКИЙ ПОДХОД
}

\begin{abstract}
Рассматривается одномерный процесс с непрерывными траекториями, обладающий однородным марковским свойством относительно момента первого выхода из любого открытого интервала (полумарковский процесс). Диффузионность процесса состоит в асимптотической равновероятности первого выхода на любую из двух границ симметричной окрестности любой начальной точки траектории при стремлении диаметра окрестности к нулю. Доказывается, что процесс имеет предел при $t \rightarrow \infty$, если вероятность невыхода из симметричной окрестности любой начальной точки траектории положительна и убывает как квадрат диаметра окрестности. Этому условию удовлетворяет, в частности, диффузионный марковский процесс с обрывом, для которого условие невыхода из данной окрестности заменено на условие обрыва до момента первого выхода из нее. Применяется полумарковский метод вывода формул условного финального распределения диффузионного процесса с пределом на бесконечности.
\end{abstract}

Ключевые слова и фразы: марковский процесс, непрерывный полумарковский процесс, марковский момент, момент первого выхода, финальная точка, условное финальное распределение.

1. Полумарковский подход. В настоящей работе рассматриваются так называемые непрерывные полумарковские процессы диффузионного типа (ПМД).

Непрерывным полумарковским процессом называется случайный процесс, обладающий однородным марковским свойством относительно момента первого выхода из любого открытого множества (см., например, [6] и [11]). В этом кратком определении подразумевается, что процесс принимает значения в топологическом пространстве и что его область определения допускает односторонние сдвиги - только в этом случае можно корректно применять термин «момент марковской регенерации», т.е. марковский момент, относительно которого выполняется однородное марковское свойство. Из этого определения следует, что каждый строго марковский процесс является непрерывным полумарковским. Очевидно также, что ступенчатые полумарковские процессы

* Институт проблем машиноведения РАН, Санкт-Петербург, Россия; e-mail: b.p.harlamov@gmail.com 
Леви-Смита [5] также удовлетворяют этому определению. Это значит, что слово «непрерывный» в названии «непрерывный полумарковский» не относится к виду выборочных траекторий, которые в данном случае могут быть как непрерывными, так и разрывными.

Класс ПМД-процессов привлекает к себе внимание как своей областью применения, так и специальным методом исследования.

Область применения ПМД-процессов - моделирование физических, биологических и социальных явлений - та же, что и у марковских диффузионных процессов (МД), которые составляют более узкий класс: МД С ПМД. Но ПМД-моделирование возможно и тогда, когда марковское свойство представляется слишком обременительным, например, когда нужно принимать в расчет замену времени, не сохраняющую марковское свойство процесса. Такая ситуация возникает, например, в хроматографии (см. [9]). При движении частиц анализируемого вещества в длинном и тонком сосуде исходный броуновский характер движения превращается в движение с остановками - интервалами постоянства, которые возникают в результате пребывания частиц на стенках сосуда. Движение происходит в результате чередования интервалов адсорбции и десорбции.

Специальная методика исследования возникла из-за невозможности использовать для немарковского процесса аппарат теории марковских процессов. Поэтому для вычисления даже стандартных функционалов приходится применять нестандартный набор полумарковских переходных функций. Однако существуют задачи, когда такой полумарковский подход оказывается полезным и для марковских процессов. Особенно наглядно польза полумарковского подхода проявляется в задачах о замедленном отражении (см. [7], [8], [10]) и вообще - в задачах о поведении процесса на границах между различными средами.

В настоящей работе рассматривается случайный процесс, выборочные траектории которого имеют предел на бесконечности. Частным случаем такого предела является так называемая бесконечная остановка интервал постоянства на бесконечности. Интерес представляет ситуация, когда не существует никакого сигнала к началу этого бесконечного интервала - он начинается спонтанно, как результат случайного выбора. Момент начала бесконечного интервала не является марковским моментом относительно натуральной фильтрации. Предыстория процесса до момента $t$ может влиять на вероятность остановки в интервале времени $[t, t+\varepsilon)$, но факт остановки не предсказывается однозначно.

Нас будет интересовать последняя точка траектории процесса до момента остановки. Эта точка фазового пространства имеет большое прикладное значение. Достаточно заметить, что в результате таких остановленных движений накапливаются горы осадочной породы на Земле. Недостаток математической модели такого физического процесса с бу- 
квальным следованием судьбе отдельной частицы (концептуальная модель) состоит в том, что она не является марковской.

В теории марковских процессов роль бесконечной остановки играет обрыв траектории процесса. Момент обрыва может быть так же непредсказуем, как момент начала бесконечной остановки. Более того, момент обрыва и положение процесса непосредственно перед обрывом могут иметь у марковского процесса то же самое распределение, что соответствующие точки у непрерывного полумарковского процесса с остановкой. В математическом смысле безразлично, по какой модели вычислять финальную точку процесса, если до момента остановки процесс развивался как диффузионный марковский процесс. Существенная разница возникла бы, если бы мы применили для решения данной математической задачи метод статистического моделирования, например если бы мы по распределению накопленной массы вещества судили о финальном распределении процесса. Тем самым, предлагается еще один вариант метода Монте-Карло для решения дифференциального уравнения.

\section{2. Непрерывный процесс с финальным распределением.}

Каждый случайный процесс рассматривается далее как случайный элемент измеримого пространства $\mathscr{D}$ функций $\xi: \mathbf{R} \rightarrow \mathbf{R}$, непрерывных справа и имеющих предел слева в каждой точке $t>0$. Предполагается, что на $\mathscr{D}$ определены метрика Скорохода и борелевская сигмаалгебра $\mathscr{F}$ подмножеств множества $\mathscr{D}$, совпадающая, как известно, с сигма-алгеброй, порожденной натуральной фильтрацией $(\mathscr{F} t)$. Количественное описание случайного процесса задается его вероятностной мерой или согласованным семейством вероятностных мер. При фиксированной мере $P$ на $(\mathscr{D}, F)$ случайный процесс может быть представлен семейством функций $\left(X_{t}\right)$, где для любого $t \geqslant 0$ и $\xi \in \mathscr{D}$ мы полагаем $X_{t}(\xi)=\xi(t)$. Это семейство функций согласовано с семейством операторов сдвига $\theta_{t}: \mathscr{D} \rightarrow \mathscr{D}$, где $\left(X_{s} \circ \theta_{t}\right)(\xi)=\xi(t+s)=X_{t+s}(\xi)$ при $s, t \geqslant 0$.

Рассмотрим одномерный случайный процесс $X(t)(t \geqslant 0)$ со значениями в некотором интервале $\mathbf{S} \subset \mathbf{R}$. Пусть $\left(P_{x}\right)(x \in \mathbf{S})$ - семейство распределений (вероятностных мер) процесса, индексированное начальными точками: $P_{x}(X(0)=x)=1$. Обозначим $\sigma_{\Delta}$ оператор первого выхода из множества $\Delta \subset \mathbf{S}$. Таким образом, рассматривается отображение $\sigma_{\Delta}: \mathscr{D} \rightarrow\left[\mathbf{R}_{+}\right]=[0, \infty]$, где

$$
\sigma_{\Delta}(\xi)=\inf \{t \geqslant 0: \xi \notin \Delta\}
$$

и, по определению, $\sigma_{\Delta}(\xi)=\infty$, если множество в фигурных скобках пусто.

Для любой точки $x \in \Delta$ могут быть определены величины $\mu_{\Delta}(x)=$ $E_{x}\left(X\left(\sigma_{\Delta}\right) ; \sigma_{\Delta}<\infty\right)$ и $E_{x}\left(f\left(X\left(\sigma_{\Delta}\right)\right) ; \sigma_{\Delta}<\infty\right)$, где $f$ - интегрируемая функция, имеющая в некоторой окрестности точки $x$ производные 
второго порядка. Предел отношения

$$
\mathscr{A}_{\Delta}(f \mid x)=\frac{E_{x}\left(f\left(X\left(\sigma_{\Delta}\right)\right) ; \sigma_{\Delta}<\infty\right)-f(x)}{\mu_{\Delta}(x)}
$$

при некотором регулярном стремлении окрестности $\Delta$ к точке $x$, если он существует, называется характеристическим оператором Дынкина (см. [2]). Характеристический оператор играет важную роль в теории МД- и ПМД-процессов.

Мы будем рассматривать процессы, для которых характеристический оператор $\mathscr{A} f$ определен на множестве дважды дифференцируемых функций и представляет собой трехчлен вида

$$
\mathscr{A}(f \mid x)=a(x) f^{\prime \prime}(x)+b(x) f^{\prime}(x)-c(x) f(x),
$$

у которого коэффициенты $a(x)$ и $c(x)$ положительны и отделены от нуля на каждом конечном интервале из области значений $[2$, гл. $17, \S 1$, п. 17.3 , c. 726].

В теории марковских процессов, где впервые появился и активно использовался термин «характеристический оператор» (см. [2, гл. 5, c. 207]), наличие члена $-c(x) f$ говорит о том, что рассматриваемый процесс $X(t)(t \geqslant 0)$ имеет обрыв в некоторый случайный момент времени $\zeta<\infty$. Это значит, что траектория процесса определяется только до момента обрыва, а сам факт обрыва вытекает из дефектности марковской переходной вероятности процесса. А именно, функция $u_{t}(x) \equiv P_{x}(X(t) \in \mathbf{R})$ (где $\mathbf{R}=(-\infty, \infty)$ - область возможных значений процесса) удовлетворяет уравнению

$$
\frac{\partial u_{t}(x)}{\partial t}=\mathscr{A}\left(u_{t} \mid x\right)
$$

откуда вытекает, что для некоторых $t>0$ и $x$

$$
P(X(t) \in \mathbf{R} \mid X(0)=x)<1
$$

Другая интерпретация данного характеристического оператора связана с классом ПМД-процессов (см. ниже). Для процессов из этого класса марковская переходная вероятность может быть определена так же, как для марковского процесса, но она не определяет меру полумарковского процесса и, самое главное, не удовлетворяет предыдущему параболическому уравнению в частных производных. Однако для ПМД-процесса существуют другие функционалы, которые удовлетворяют родственному уравнению эллиптического типа. В частности, функционал

$$
f_{\Delta}(x) \equiv P_{x}\left(\sigma_{\Delta}<\infty\right)
$$


(где $\Delta \subset \mathbf{S})$ удовлетворяет уравнению

$$
\mathscr{A}\left(f_{\Delta} \mid x\right)=0 \quad(x \in \Delta)
$$

принимая значения 1 в краевых точках интервала. Отсюда следует, что $f_{\Delta}(x)<1$ внутри интервала. И если допустить, что траектории процесса не обрываются, то для некоторых точек $x \in \Delta$ должно выполняться условие

$$
P_{x}\left(\sigma_{\Delta}=\infty\right)>0
$$

А это приводит к совершенно иной интерпретации данного характеристического оператора. Вместо процесса с обрывом появляется процесс с остановкой - бесконечным интервалом постоянства в конце траектории. Доказательству этого факта и выводу предельного (финального) распределения для такого процесса посвящена настоящая статья.

Непрерывный процесс с пределом на бесконечности. Итак, мы делаем предположение, что наш процесс принадлежит более широкому классу, чем класс строго марковских процессов. Заметим, что во всех прочих отношениях полумарковский диффузионный процесс может быть подобен марковскому диффузионному процессу. Только в предполагаемый момент обрыва процесс не обрывается, а останавливается навсегда в достигнутой к этому моменту точке своей траектории. Это отличие имеет принципиальное значение. Пусть $\zeta$ - момент остановкиобрыва и $X(\zeta-)$ - точка остановки-обрыва (для обрыва - это точка непосредственно перед обрывом, для остановки - это именно точка остановки $X(\zeta-)=X(t)$ для всех $t \geqslant \zeta)$. Момент $\zeta$ не является марковским моментом ни относительно $(\mathscr{F})_{t}$, ни относительно $(\mathscr{F})_{t+}$, так как информация о начале некоторого интервала постоянства, получаемая до момента $t+\varepsilon(\varepsilon>0)$, не означает, что этот интервал бесконечен. Особенно это актуально для не марковского ПМД-процесса, имеющего конечные интервалы постоянства до момента окончательной остановки. $\mathrm{C}$ другой стороны, момент первого достижения точки $X(\zeta-)$ не обязан быть началом бесконечного интервала постоянства (ПМД-процесс может много раз достигать этой точки, не останавливаясь в ней). Это дает основание утверждать, что процесс с бесконечной остановкой не является марковским процессом (за исключением тривиальных случаев).

Решающий довод против использования процесса с обрывом в качестве модели реального явления дает задача о накоплении вещества. В случае процесса с остановкой финальное распределение одной частицы определяет распределение массы, составленной из независимых частиц с одинаковым законом движения. В случае обрыва мера накопленной массы равна нулю.

Рассмотрим одномерный непрерывный случайный процесс $X(t)$ $(t \geqslant 0)$. Распределение $P_{x}$ процесса с начальным состоянием $x \in \mathbf{R}$ 
представляет собой вероятностную меру на измеримом пространстве $(\mathscr{C}, \mathscr{F})$, где $\mathscr{C}$ - множество всех непрерывных выборочных функций $\xi:[0, \infty) \rightarrow \mathbf{R}$, a $\mathscr{F}$ - борелевская сигма-алгебра подмножеств этого множества (как подмножества $\mathscr{D}$ ). В дальнейшем мы будем использовать специальное обозначение для события, состоящего в том, что процесс с некоторого момента не выходит из интервала $\Delta$. Обозначим

$$
\rho(\Delta) \equiv \bigcup_{t \geqslant 0} \theta_{t}^{-1}\left(\sigma_{\Delta}=\infty\right)
$$

Пусть $\left(\tau_{n}\right)$ - последовательность случайных величин, стремящаяся почти наверное к бесконечности при $n \rightarrow \infty$. Тогда с вероятностью единица справедливо равенство

$$
\rho(\Delta)=\bigcup_{n=1}^{\infty} \theta_{\tau_{n}}^{-1}\left(\sigma_{\Delta}=\infty\right) .
$$

Ясно, что $\left\{\sigma_{\Delta}=\infty\right\} \subset \rho(\Delta)$.

Обозначим $\mathscr{C}^{\text {lim }}$ множество непрерывных выборочных траекторий $\xi$, имеющих предел на бесконечности. Одной из причин, по которой процесс с некоторого момента времени не выходит из интервала, является выполнение условия «существует $\lim _{t \rightarrow \infty} X(t)$, принадлежащий этому интервалу».

Лемма 1. На множестве $\mathscr{C}$ справедливо соотношение

$$
\mathscr{C}^{\lim } \cap\left\{\sigma_{\Delta}=\infty\right\}=\bigcap_{\varepsilon>0} \bigcup_{\Delta_{1} \in \Pi(\varepsilon)} \rho\left(\Delta_{1}\right)
$$

где $\Delta$ - открьтыий интервал и $(\varepsilon)$ - покрьтие множества $\Delta$ открытылми интервалами длиной не более $\varepsilon$, где $\bigcup_{\Delta_{1} \in \Pi(\varepsilon)} \Delta_{1}=\Delta$.

Д о к а з а т е л с т в о. Каждая функция $\xi \in \bigcap_{\varepsilon>0} \bigcup_{\Delta \in \Pi_{\varepsilon}} \rho(\Delta)$ удовлетворяет критерию Коши на бесконечности и поэтому сходится (сходится в себе). Обратное включение тривиально. Лемма доказана.

Множеству процессов с траекториями из $\mathscr{C}^{\text {lim }}$ в качестве специального подкласса принадлежат процессы, имеющие так называемую бесконечную остановку - бесконечный интервал постоянства на своей области определения. Легко определить начало бесконечной остановки, располагая информацией о всей траектории в целом. Но нельзя узнать по предыстории процесса до любого конечного момента времени, что бесконечная остановка уже началась. Это означает, что начало бесконечной остановки (случайный момент $\zeta$ ) является измеримой функцией относительно $\mathscr{F}$, но не является марковским моментом относительно натуральной фильтрации. 
Обозначим $\mathscr{C}^{\infty}$ множество выборочных траекторий, имеющих бесконечную остановку. По определению, $\mathscr{C}^{\infty} \subset \mathscr{C}^{\mathrm{lim}}$. Нетрудно доказать, что оба эти множества принадлежат $\mathscr{F}$.

В задаче о финальном распределении, решаемой полумарковским методом, отличие процессов с бесконечной остановкой от процессов, имеющих предел общего вида на бесконечности, не представляется существенным до тех пор, пока не ставится задача о начале бесконечной остановки. В данной работе мы не будем изучать распределение этого момента.

Непрерывный полумарковский процесс. Пусть $\left(P_{x}\right)$ - измеримое согласованное семейство мер, индексированных начальными точками траектории процесса $x \in \mathbf{R}$. Мы предположим, что согласование мер семейства $\left(P_{x}\right)$ определяется условием:

$$
P_{x}\left(\theta_{\sigma_{\Delta}}^{-1} B, A, \sigma_{\Delta}<\infty\right)=E_{x}\left(P_{X\left(\sigma_{\Delta}\right)}(B) ; A, \sigma_{\Delta}<\infty\right)
$$

для всех $x \in \mathbf{R}, B \in \mathscr{F}$ и $A \in \mathscr{F}_{\sigma_{\Delta}}$, где $\Delta$ - интервал из множества значений процесса, $\mathscr{F}_{\sigma_{\Delta}}$ - сигма-алгебра, определяемая стандартным образом относительно марковского момента $\sigma_{\Delta}$. Таким образом, вероятность, зависящая от значения процесса в случайный момент $\sigma_{\Delta}$, почти наверное совпадает с условной вероятностью относительно всей предыстории процесса до этого момента, т.е. выполняется однородное марковское условие в момент первого выхода из открытого интервала, включенного в область значений процесса [6]. В общем случае этот процесс не является марковским. Такой процесс называется непрерывным полумарковским [6]. Прилагательное «непрерывный» используется для отличия от того определения, которое дается в теории ступенчатых полумарковских процессов (хотя определение непрерывного полумарковского процесса обслуживает и ступенчатые процессы). Заметим, что согласно этому определению каждый строго марковский процесс является непрерывным полумарковским процессом, но не наоборот.

Для каждого интервала $(a, b)$ и $x \in(a, b)$ определим полумарковские переходные функции:

$$
\begin{aligned}
& G_{(a, b)}(d t \mid x) \equiv P_{x}\left(\sigma_{(a, b)} \in d t, X\left(\sigma_{(a, b)}\right)=a\right) \\
& H_{(a, b)}(d t \mid x) \equiv P_{x}\left(\sigma_{(a, b)} \in d t, X\left(\sigma_{(a, b)}\right)=b\right)
\end{aligned}
$$

и их преобразования Лапласа по $t(\lambda \geqslant 0)$ :

$$
\begin{aligned}
& g_{(a, b)}(\lambda, x) \equiv \int_{0}^{\infty} e^{-\lambda t} G_{(a, b)}(d t \mid x), \\
& h_{(a, b)}(\lambda, x) \equiv \int_{0}^{\infty} e^{-\lambda t} H_{(a, b)}(d t \mid x) .
\end{aligned}
$$


Таким образом,

$$
\begin{aligned}
& g_{(a, b)}(\lambda, x)=E_{x}\left(\exp \left(-\lambda \sigma_{(a, b)}\right) ; \sigma_{(a, b)}<\infty, X\left(\sigma_{(a, b)}\right)=a\right), \\
& h_{(a, b)}(\lambda, x)=E_{x}\left(\exp \left(-\lambda \sigma_{(a, b)}\right) ; \sigma_{(a, b)}<\infty, X\left(\sigma_{(a, b)}\right)=b\right) .
\end{aligned}
$$

Мы предположим, что для предельных значений выполняются условия

$$
g_{(a, b)}(\lambda, a+)=h_{(a, b)}(\lambda, b-)=1, \quad g_{(a, b)}(\lambda, b-)=h_{(a, b)}(\lambda, a+)=0,
$$

а в граничных точках интервала значения этих функций определяются по непрерывности.

Определим сумму «со сдвигом» для двух неотрицательных функций $\tau_{1}$ и $\tau_{2}$ на $\mathscr{D}$ (могущих принимать бесконечное значение) следующим образом:

$$
\tau_{1} \dot{+} \tau_{2}=\tau_{1}+\tau_{2} \circ \theta_{\tau_{1}}
$$

на множестве $\left\{\tau_{1}<\infty\right\}$ и $\tau_{1} \dot{+} \tau_{2}=\infty$ на множестве $\left\{\tau_{1}=\infty\right\}$. Операция $\dot{+}$ обладает свойством ассоциативности, но не коммутативна.

Известно, что для любого интервала $(c, d)$ такого, что $(c, d) \subset(a, b)$, выполняется равенство

$$
\sigma_{(a, b)}=\sigma_{(c, d)} \dot{+} \sigma_{(a, b)} .
$$

Из этого соотношения, из определенных выше значений функций $g_{(c, d)}$ и $h_{(c, d)}$ на концах интервала $(c, d)$ и из полумарковского свойства процесса следуют уравнения (см. [6])

$$
\begin{aligned}
& g_{(a, b)}(\lambda, x)=g_{(c, d)}(\lambda, x) g_{(a, b)}(\lambda, c)+h_{(c, d)}(\lambda, x) g_{(a, b)}(\lambda, d), \\
& h_{(a, b)}(\lambda, x)=g_{(c, d)}(\lambda, x) h_{(a, b)}(\lambda, c)+h_{(c, d)}(\lambda, x) h_{(a, b)}(\lambda, d)
\end{aligned}
$$

для любой точки $x$ такой, что $x \in[c, d] \subset[a, b]$, и $\lambda \geqslant 0$.

Мы будем иметь дело с процессами, для которых существуют интервалы значений $(a, b)$ и точки $x \in(a, b)$ такие, что $P_{x}\left(\sigma_{(a, b)}=\infty\right)>0$. Заметим, что если для данного $x$ эта вероятность меньше единицы, то может случиться, что процесс много раз выходит из интервала $(a, b)$ и возвращается в него, пока не останется в этом интервале навсегда после одного такого возвращения. В этом состоит смысл события (подмножества) $\rho(\Delta)$, когда $\Delta=(a, b)$.

Полумарковский диффузионный процесс. Полумарковский процесс называется диффузионным в окрестности точки $x$, если существуют функции $A(x)$ и $B(\lambda, x)$ такие, что

$$
\begin{aligned}
& g_{(x-r, x+r)}(\lambda, x)=\frac{1}{2}\left(1-A(x) r-B(\lambda, x) r^{2}\right)+o\left(r^{2}\right), \\
& h_{(x-r, x+r)}(\lambda, x)=\frac{1}{2}\left(1+A(x) r-B(\lambda, x) r^{2}\right)+o\left(r^{2}\right)
\end{aligned}
$$


при $r \rightarrow 0$. Предполагается, что функция $A(x)$ непрерывно дифференцируема в окрестности точки $x$, а функция $B(\lambda, x)$ положительна, непрерывна по второму аргументу в окрестности точки $x$, не убывает и имеет вполне монотонную частную производную по первому аргументу. Если условие диффузионности выполняется для любой точки открытого интервала $(a, b)$ при некоторых допустимых функциях $A(x), B(\lambda, x)$ $(x \in(a, b), \lambda \geqslant 0)$, то функции $g_{(a, b)}(\lambda, x), h_{(a, b)}(\lambda, x)$ удовлетворяют на этом интервале дифференциальному уравнению (см. [6])

$$
\frac{1}{2} f^{\prime \prime}+A(x) f^{\prime}-B(\lambda, x) f=0
$$

с краевыми значениями

$$
g_{(a, b)}(\lambda, a)=h_{(a, b)}(\lambda, b)=1, \quad g_{(a, b)}(\lambda, b)=h_{(a, b)}(\lambda, a)=0
$$

и, следовательно, при любом $\lambda \geqslant 0$ составляют фундаментальную систему решений этого уравнения.

Для нас особый интерес представляют диффузионные полумарковские процессы, у которых $B(0, x)>0$ при любом $x \in(a, b)$ и эта функция отделена от нуля на любом конечном интервале. Именно для таких коэффициентов при $\lambda=0$ уравнение (6) имеет решения, подчиняющиеся условию $g_{(c, d)}(0, x)+h_{(c, d)}(0, x)<1$ при любых $(c, d) \subset[a, b]$ и $x \in(c, d)$, которое эквивалентно условию $P_{x}\left(\sigma_{(c, d)}=\infty\right)>0$.

Марковские диффузионные процессы составляют подкласс класса полумарковских диффузионных процессов. Марковость полумарковского диффузионного процесса может быть определена в терминах коэффициентов уравнения (6). Полумарковский диффузионный процесс является локально марковским на интервале $(a, b)$, если существует функция $B_{0}(x)>0$ такая, что для любых $x \in(a, b)$ и $\lambda \geqslant 0$ справедливо представление

$$
B(\lambda, x)=\lambda B_{0}(x) .
$$

В этом случае коэффициенты уравнения могут быть выражены через феллеровские диффузионные коэффициенты $b(x)$ (снос) и $a(x)$ (локальная дисперсия), а именно, $A(x)=b(x) / a(x), B_{0}(x)=1 / a(x)[6]$.

3. Предел на бесконечности. Пусть

$$
\begin{aligned}
& G_{(a, b)}(x) \equiv g_{(a, b)}(0, x)=P_{x}\left(\sigma_{(a, b)}<\infty, X\left(\sigma_{(a, b)}\right)=a\right), \\
& H_{(a, b)}(x) \equiv h_{(a, b)}(0, x)=P_{x}\left(\sigma_{(a, b)}<\infty, X\left(\sigma_{(a, b)}\right)=b\right)
\end{aligned}
$$

- маргинальные переходные вероятности, где $-\infty<a<x<b<\infty$. Эти функции на интервале $(a, b)$ удовлетворяют уравнению

$$
\frac{1}{2} f^{\prime \prime}+A(x) f^{\prime}-B(0, x) f=0
$$


(далее будем обозначать $B(0, x) \equiv B(x))$ с краевыми значениями $G_{(a, b)}(a)=H_{(a, b)}(b)=1$ и $G_{(a, b)}(b)=H_{(a, b)}(a)=0$. Решение задачи о финальном распределении процесса мы дадим в терминах этих функций.

Теорема 1. Если всюду на замкнутом интервале $[a, b]$ непрерывная функиия $B(x)$ положительна, то для любой начальной точки $x \in$ $\Delta \equiv(a, b)$ справедливь соотношения $P_{x}\left(\sigma_{\Delta}=\infty\right)>0 u$

$$
P_{x}\left(\mathscr{C}^{\lim } \mid \sigma_{\Delta}=\infty\right)=1 \text {. }
$$

Д о к а з а т е л ь с т в о. В условии теоремы рассматривается процесс, для которого при любых $(c, d) \subset[a, b]$ и $x \in(c, d)$ справедливо неравенство $P_{x}\left(\sigma_{(c, d)}=\infty\right) \equiv 1-G_{(c, d)}(x)-H_{(c, d)}(x)>0$. Пусть $a=a_{1}<a_{2}<b_{1}<b_{2}=b$. Обозначим

$$
\Delta_{i}=\left(a_{i}, b_{i}\right) \quad(i=1,2) .
$$

Далее, наряду с обозначением $\kappa(\Delta)=\{\sigma(\Delta)=\infty\}$ при $\Delta=(a, b)$ введем обозначения

$$
\begin{aligned}
& \kappa_{1}(\Delta)=\{\sigma(\Delta)<\infty, X(\sigma(\Delta))=a\}, \\
& \kappa_{2}(\Delta)=\{\sigma(\Delta)<\infty, X(\sigma(\Delta))=b\} .
\end{aligned}
$$

В дальнейшем, записывая соотношения между множествами, мы будем заменять знак $\cup$ (дизъюнкции) знаком + , если компоненты объединения не пересекаются, а также опускать знак пересечения $\cap$ (конъюнкции) или заменять его точкой там, где это не вызывает двойного толкования. Пусть $x \in \Delta_{1}$. Это определяет первое представление события $\kappa(\Delta)$ и порядок следования точек первого выхода. Другое расположение начальной точки внутри интервала $\Delta$ рассматривается аналогично.

Для первого представления обозначим $\tau_{0}=0, \tau_{1}=\sigma_{\Delta_{1}}, \tau_{2}=\tau_{1} \dot{+} \sigma_{\Delta_{2}}$,

$$
\tau_{2 n+1}=\tau_{2 n} \dot{+} \sigma_{\Delta_{1}}, \quad \tau_{2 n+2}=\tau_{2 n+1} \dot{+} \sigma_{\Delta_{2}} \quad n \geqslant 0 .
$$

Пусть

$$
\begin{gathered}
B_{0}=\kappa_{2}\left(\Delta_{1}\right), \quad B_{1}=\theta_{\tau_{1}}^{-1} \kappa_{1}\left(\Delta_{2}\right), \quad B_{2}=\theta_{\tau_{2}}^{-1} \kappa_{2}\left(\Delta_{1}\right), \\
B_{2 n+1}=\theta_{\tau_{2 n+1}}^{-1} \kappa_{1}\left(\Delta_{2}\right), \quad B_{2 n+2}=\theta_{\tau_{2 n+2}}^{-1} \kappa_{2}\left(\Delta_{1}\right) \quad(n \geqslant 0), \\
A_{0}=\kappa\left(\Delta_{1}\right), \quad A_{1}=A_{0}+B_{0} \theta_{\tau_{1}}^{-1} \kappa\left(\Delta_{2}\right), \quad A_{2}=A_{1}+B_{0} B_{1} \theta_{\tau_{2}}^{-1} \kappa\left(\Delta_{1}\right), \\
A_{2 n+1}=A_{2 n}+B_{0} B_{1} \cdots B_{2 n} \theta_{\tau_{2 n+1}}^{-1} \kappa\left(\Delta_{2}\right) \quad(n \geqslant 0), \\
A_{2 n+2}=A_{2 n+1}+B_{0} B_{1} \cdots B_{2 n+1} \theta_{\tau_{2 n+2}}^{-1} \kappa\left(\Delta_{1}\right) \quad(n \geqslant 0) .
\end{gathered}
$$

В этих терминах мы составляем уравнения — последовательные ступени разложения в ряд для множества $\kappa(\Delta)$. Имеем

$$
\kappa(\Delta)=A_{0}+B_{0} \theta_{\tau_{1}}^{-1} \kappa(\Delta) .
$$


Подставляя в правую часть этого равенства представление $\kappa(\Delta)$ относительно начальной точки, принадлежащей множеству $\Delta_{2}$, и используя распределительное свойство обратного отображения сдвига $\theta_{t}^{-1}$ и свойство ассоциативности оператора сдвига $\theta_{\tau_{1}}^{-1} \theta_{\tau_{2}}^{-1}=\theta_{\tau_{1}+\tau_{2}}^{-1}$, получаем

$$
\kappa(\Delta)=A_{0}+B_{0} \theta_{\tau_{1}}^{-1}\left[\kappa\left(\Delta_{2}\right)+\kappa_{1}\left(\Delta_{2}\right) \theta_{\sigma\left(\Delta_{2}\right)}^{-1} \kappa(\Delta)\right]=A_{1}+B_{0} B_{1} \theta_{\tau_{2}}^{-1} \kappa(\Delta) .
$$

Продолжая этот процесс, по индукции получаем формулу

$$
\kappa(\Delta)=A_{n}+B_{0} B_{1} \cdots B_{n} \theta_{\tau_{n+1}}^{-1} \kappa(\Delta) \quad(n \geqslant 0) .
$$

Последовательность множеств $\left(A_{n}\right)$ монотонно возрастает, последовательность остатков

$$
B_{0} B_{1} \cdots B_{n} \theta_{\tau_{n+1}}^{-1} \kappa(\Delta) \quad(n \geqslant 0)
$$

монотонно убывает. Имеем

$$
\begin{aligned}
P_{x}\left(B_{0} B_{1} \cdots B_{n} \theta_{\tau_{n+1}}^{-1} \kappa(\Delta)\right) \\
=\left\{\begin{array}{c}
H_{\Delta_{1}}(x)\left[G_{\Delta_{2}}\left(b_{1}\right) H_{\Delta_{1}}\left(a_{2}\right)\right]^{n / 2} G_{\Delta_{2}}\left(b_{1}\right)\left(1-G_{\Delta}\left(a_{2}\right)-H_{\Delta}\left(a_{2}\right)\right), \\
n-\text { четное; } \\
H_{\Delta_{1}}(x)\left[G_{\Delta_{2}}\left(b_{1}\right) H_{\Delta_{1}}\left(a_{2}\right)\right]^{(n+1) / 2}\left(1-G_{\Delta}\left(b_{1}\right)-H_{\Delta}\left(b_{1}\right)\right), \\
n-\text { нечетное },
\end{array}\right.
\end{aligned}
$$

Эта вероятность стремится к нулю при $n \rightarrow \infty$. Поэтому последовательность множеств $\left(A_{n}\right)$ стремится к пределу $\kappa(\Delta)$ с $P_{x}$-вероятностью 1. Справедливо следующее равенство: $P_{x}$-п.н.

$$
\kappa(\Delta)=\kappa\left(\Delta_{1}\right)+\sum_{n=1}^{\infty} B_{0} B_{1} \cdots B_{n-1} \theta_{\tau_{n}}^{-1} \begin{cases}\kappa\left(\Delta_{1}\right), & n \text { - четное; } \\ \kappa\left(\Delta_{2}\right), & n \text { - нечетное. }\end{cases}
$$

Перегруппируем правую часть этого равенства:

$$
\begin{aligned}
\kappa(\Delta)= & \left(\kappa\left(\Delta_{1}\right)+\sum_{n=1}^{\infty} B_{0} B_{1} \cdots B_{2 n-1} \cdot \theta_{\tau_{2 n}}^{-1} \kappa\left(\Delta_{1}\right)\right) \\
& +\sum_{n=0}^{\infty} B_{0} B_{1} \cdots B_{2 n} \cdot \theta_{\tau_{2 n+1}}^{-1} \kappa\left(\Delta_{2}\right) .
\end{aligned}
$$

Убирая из правой части компоненты $B_{i}$, мы можем ее только увеличить. Отсюда следует п.н. равенство

$$
\kappa(\Delta)=\kappa(\Delta)\left(\bigcup_{n=0}^{\infty} \theta_{\tau_{2 n}}^{-1} \kappa\left(\Delta_{1}\right) \cup \bigcup_{n=0}^{\infty} \theta_{\tau_{2 n+1}}^{-1} \kappa\left(\Delta_{2}\right)\right) .
$$


При данном расположении концов перекрывающихся интервалов последовательность $\left(\tau_{n}\right)$ стремится к бесконечности $P_{x}$-П.н. Следовательно, справедливо равенство

$$
\kappa(\Delta)=\kappa(\Delta)\left[\rho\left(\Delta_{1}\right) \cup \rho\left(\Delta_{2}\right)\right]
$$

откуда вытекает равенство

$$
P_{x}\left(\rho\left(\Delta_{1}\right) \cup \rho\left(\Delta_{2}\right) \mid \kappa(\Delta)\right)=1 .
$$

С другой стороны,

$$
\begin{aligned}
\rho(\Delta) & =\bigcup_{t \geqslant 0} \theta_{t}^{-1} \kappa(\Delta) \subset \bigcup_{t \geqslant 0} \theta_{t}^{-1}\left(\rho\left(\Delta_{1}\right) \cup \rho\left(\Delta_{2}\right)\right) \\
& =\bigcup_{t \geqslant 0}\left(\rho\left(\Delta_{1}\right) \cup \rho\left(\Delta_{2}\right)\right)=\rho\left(\Delta_{1}\right) \cup \rho\left(\Delta_{2}\right) .
\end{aligned}
$$

Обратное включение тривиально.

Рассмотрим теперь конечное покрытие $\gamma_{n}=\left(\Delta_{k}\right)_{1}^{n}$ интервала $(a, b)$ интервалами $\Delta_{k}$ вида

$$
\begin{gathered}
\Delta_{k}=\left(a_{k}, b_{k}\right), \quad 1 \leqslant k \leqslant n, \quad n \geqslant 2, \quad \bigcup_{k=1}^{n} \Delta_{k}=(a, b), \\
a=a_{1}<a_{2}<b_{1}<a_{3}<b_{2}<a_{4}<b_{3}<a_{5}<b_{4}<\cdots<b_{n-1}<b_{n}=b .
\end{gathered}
$$

Применяя полученную формулу к подпокрытиям этого покрытия, приходим к формуле

$$
\kappa(\Delta)=\kappa(\Delta) \bigcup_{k=1}^{n} \rho\left(\Delta_{k}\right)
$$

Ясно, что таким путем можно получить покрытие $\gamma_{n}$ со сколь угодно мелкими элементами. Пусть $\varepsilon_{n}$ - максимальная длина элементов покрытия $\gamma_{n}$ и $\varepsilon_{n} \rightarrow 0$. Из доказанной выше формулы следует, что

$$
P_{x}\left(\bigcup_{\Delta_{k} \in \gamma_{n}}^{n} \rho\left(\Delta_{k}\right) \mid \sigma_{\Delta}=\infty\right)=1 .
$$

Отсюда для любого $N \geqslant 1$ вытекает равенство

$$
P_{x}\left(\bigcap_{n=1}^{N} \bigcup_{\Delta_{k} \in \gamma_{n}}^{n} \rho\left(\Delta_{k}\right) \mid \sigma_{\Delta}=\infty\right)=1,
$$

и, следовательно, по лемме 1

$$
P_{x}\left(\mathscr{C}^{\lim } \mid \sigma_{\Delta}=\infty\right)=1
$$

т.е. процесс имеет предел на бесконечности с условной вероятностью 1. Теорема 1 доказана. 
4. Финальное распределение. Процесс, имеющий предел на бесконечности, не является эргодическим, но для него при любых $x \in(a, b)$ существует предельное одномерное распределение (финальное распределение)

$$
Q_{(a, b)}(y \mid x) \equiv P_{x}\left(\lim _{t \rightarrow \infty} X(t)<y \mid \sigma_{(a, b)}=\infty\right),
$$

где $a<y \leqslant b$. Это ядро характеризует условное распределение финальной точки $X(\zeta)$, когда переход к пределу или остановка процесса произошла до момента первого выхода из интервала $(a, b)$. Распределение $Q_{(a, b)}(y \mid x)$ может быть найдено непосредственно в терминах маргинальных переходных вероятностей.

Мы применяем полумарковский метод вывода формул условного финального распределения ПМД-процесса.

Теорема 2. Пусть выполняются условия теоремь 1. Тогда:

1) если $x<y$, то

$$
Q_{\Delta}(y \mid x)=\left(1-G_{\Delta}(x)-H_{\Delta}(x)\right)^{-1}\left(1-G_{\Delta}(x)+H_{\Delta}(x) \frac{G_{\Delta}^{\prime}(y)}{W_{\Delta}(y)}\right)
$$

2) если $x>y$, mо

$$
Q_{\Delta}(y \mid x)=\left(1-G_{\Delta}(x)-H_{\Delta}(x)\right)^{-1}\left(-G_{\Delta}(x)+G_{\Delta}(x) \frac{H_{\Delta}^{\prime}(y)}{W_{\Delta}(y)}\right)
$$

здесь $W_{\Delta}(y)=G_{\Delta}(y) H_{\Delta}^{\prime}(y)-H_{\Delta}(y) G_{\Delta}^{\prime}(y)$ - вронскиан (см., например, [4, ч. 1 , п. 17.1 , с. 95]).

Доказ а т ельст в о. 1) Пусть $a=a_{1}<a_{2}<b_{1}<b_{2}=b$, $\Delta=(a, b), \Delta_{1}=\left(a_{1}, b_{1}\right), \Delta_{2}=\left(a_{2}, b_{2}\right), x \in \Delta_{1}$. Рассмотрим процесс, начинающийся из точки $x$. При доказательстве теоремы 1 мы вывели формулу

$$
\kappa(\Delta)=\Psi_{1}+\Psi_{2}
$$

где

$$
\Psi_{1}=\kappa\left(\Delta_{1}\right)+\sum_{n=1}^{\infty} B_{0} B_{1} \cdots B_{2 n-1} \theta_{\tau_{2 n}}^{-1} \kappa\left(\Delta_{1}\right)
$$

- множество траекторий, начинающихся в $\Delta_{1}$ и не выходящих из $\Delta$ (они или не выходят из $\Delta_{1}$, или выходят из $\Delta_{1}$, попадая в $\Delta_{2}$, и выходят из интервала $\Delta_{2}$, попадая в интервал $\Delta_{1}$, как в начальное состояние), и

$$
\Psi_{2}=\sum_{n=0}^{\infty} B_{0} B_{1} \cdots B_{2 n} \cdot \theta_{\tau_{2 n+1}}^{-1} \kappa\left(\Delta_{2}\right)
$$

- множество траекторий, начинающихся в $\Delta_{2} \backslash \Delta_{1}$ и не выходящих из $\Delta$ (они или не выходят из $\Delta_{2}$, или выходят из $\Delta_{2}$, попадая в $\Delta_{1}$, и выходят из интервала $\Delta_{1}$, попадая в интервал $\Delta_{2}$, как в начальное состояние). 
Каждая траектория, не выходящая из $\Delta$, или остается навсегда на конечном шаге в одном из интервалов $\Delta_{1}$ или $\Delta_{2}$, или бесконечное число раз переходит из одного интервала в другой - вероятность этого исхода равна нулю.

Имеем $\Psi_{1} \subset\left\{\lim _{t \rightarrow \infty} X(t) \leqslant b_{1}\right\}$ и $\Psi_{2} \subset\left\{\lim _{t \rightarrow \infty} X(t) \geqslant a_{2}\right\}$. Отсюда

$$
\begin{aligned}
P_{x}\left(\Psi_{1} \mid \sigma_{\Delta}=\infty\right) & \leqslant P_{x}\left(\lim _{t \rightarrow \infty} X(t) \leqslant b_{1} \mid \sigma_{\Delta}=\infty\right), \\
P_{x}\left(\Psi_{2} \mid \sigma_{\Delta}=\infty\right) & =1-P_{x}\left(\Psi_{1} \mid \sigma_{\Delta}=\infty\right) \leqslant P_{x}\left(\lim _{t \rightarrow \infty} X(t) \geqslant a_{2} \mid \sigma_{\Delta}=\infty\right) \\
& =1-P_{x}\left(\lim _{t \rightarrow \infty} X(t)<a_{2} \mid \sigma_{\Delta}=\infty\right) .
\end{aligned}
$$

Следовательно,

$$
\begin{gathered}
P_{x}\left(\lim _{t \rightarrow \infty} X(t)<a_{2} \mid \sigma_{\Delta}=\infty\right) \leqslant P_{x}\left(\Psi_{1} \mid \sigma_{\Delta}=\infty\right) \\
\leqslant P_{x}\left(\lim _{t \rightarrow \infty} X(t) \leqslant b_{1} \mid \sigma_{\Delta}=\infty\right)
\end{gathered}
$$

и при $a_{2} \rightarrow b_{1}$ левая часть двойного неравенства стремится к правой части в любой точке непрерывности условной функции распределения случайной величины $\lim _{t \rightarrow \infty} X(t)$. Отсюда следует, что для почти всех $b_{1}$ (по мере Лебега)

$$
P_{x}\left(\lim _{t \rightarrow \infty} X(t) \leqslant b_{1} \mid \sigma_{\Delta}=\infty\right)=\lim _{a_{2} \rightarrow b_{1}} P_{x}\left(\Psi_{1} \mid \sigma_{\Delta}=\infty\right) .
$$

Имеем

$$
\begin{aligned}
P_{x}\left(\Psi_{1}\right)= & P_{x}\left(\kappa\left(\Delta_{1}\right)+\sum_{n=1}^{\infty} B_{0} B_{1} \cdots B_{2 n-1} \cdot \theta_{\tau_{2 n}}^{-1} \kappa\left(\Delta_{1}\right)\right) \\
= & P_{x}\left(\kappa\left(\Delta_{1}\right)\right)+\sum_{n=1}^{\infty} P_{x}\left(B_{0} B_{1} \cdots B_{2 n-1} \cdot \theta_{\tau_{2 n}}^{-1} \kappa\left(\Delta_{1}\right)\right) \\
= & 1-G_{\Delta_{1}}(x)-H_{\Delta_{1}}(x)+H_{\Delta_{1}}(x) G_{\Delta_{2}}\left(b_{1}\right)\left(1-G_{\Delta_{1}}\left(a_{2}\right)-H_{\Delta_{1}}\left(a_{2}\right)\right) \\
& +\sum_{n=1}^{\infty} H_{\Delta_{1}}(x)\left(G_{\Delta_{2}}\left(b_{1}\right) H_{\Delta_{1}}\left(a_{2}\right)\right)^{n} G_{\Delta_{2}}\left(b_{1}\right)\left(1-G_{\Delta_{1}}\left(a_{2}\right)-H_{\Delta_{1}}\left(a_{2}\right)\right) \\
= & 1-G_{\Delta_{1}}(x)-H_{\Delta_{1}}(x)+H_{\Delta_{1}}(x) G_{\Delta_{2}}\left(b_{1}\right) \frac{1-G_{\Delta_{1}}\left(a_{2}\right)-H_{\Delta_{1}}\left(a_{2}\right)}{1-G_{\Delta_{2}}\left(b_{1}\right) H_{\Delta_{1}}\left(a_{2}\right)} .
\end{aligned}
$$

Найдем предел этого выражения при $a_{2} \rightarrow b_{1} \equiv y$. Обозначим $a_{2}=$ $y-h$. Имеем $H_{\Delta}(y-h)=H_{\Delta_{1}}(y-h) H_{\Delta}(y)$. Отсюда по формуле Тейлора получаем

$$
H_{\Delta_{1}}(y-h)=\frac{H_{\Delta}(y-h)}{H_{\Delta}(y)}=1-\frac{H_{\Delta}^{\prime}(y)}{H_{\Delta}(y)} h+o(h) .
$$


Далее, $G_{\Delta}(y-h)=G_{\Delta_{1}}(y-h)+H_{\Delta_{1}}(y-h) G_{\Delta}(y)$, откуда

$$
\begin{aligned}
G_{\Delta_{1}}(y-h) & =G_{\Delta}(y-h)-H_{\Delta_{1}}(y-h) G_{\Delta}(y) \\
& =G_{\Delta}(y)-G_{\Delta}^{\prime}(y) h+o(h)-G_{\Delta}(y)\left(1-\frac{H_{\Delta}^{\prime}(y)}{H_{\Delta}(y)} h+o(h)\right) \\
& =\frac{W_{\Delta}(y)}{H_{\Delta}(y)} h+o(h) .
\end{aligned}
$$

Кроме того, $G_{\Delta}(y)=G_{\Delta_{2}}(y) G_{\Delta}(y-h)$, и, значит,

$$
G_{\Delta_{2}}(y)=\frac{G_{\Delta}(y)}{G_{\Delta}(y-h)}=\frac{G_{\Delta}(y)}{G_{\Delta}(y)-G_{\Delta}^{\prime}(y) h+o(h)}=1+\frac{G_{\Delta}^{\prime}(y)}{G_{\Delta}(y)} h+o(h) .
$$

Следовательно,

$$
\begin{aligned}
& P_{x}\left(\Psi_{1}\right)=1-G_{\Delta_{1}}(x)-H_{\Delta_{1}}(x)+H_{\Delta_{1}}(x)\left(1+\frac{G_{\Delta}^{\prime}(y)}{G_{\Delta}(y)} h+o(h)\right) \\
& \times\left(1-\frac{W_{\Delta}(y)}{H_{\Delta}(y)} h+o(h)-\left(1-\frac{H_{\Delta}^{\prime}(y)}{H_{\Delta}(y)} h+o(h)\right)\right) \\
& \times\left(1-\left(1+\frac{G_{\Delta}^{\prime}(y)}{G_{\Delta}(y)} h+o(h)\right)\left(1-\frac{H_{\Delta}^{\prime}(y)}{H_{\Delta}(y)} h+o(h)\right)\right)^{-1} \\
& =1-G_{\Delta_{1}}(x)-H_{\Delta_{1}}(x)+H_{\Delta_{1}}(x)\left(-\frac{W_{\Delta}(y)}{H_{\Delta}(y)} h+\frac{H_{\Delta}^{\prime}(y)}{H_{\Delta}(y)} h+o(h)\right) \\
& \left.\times\left(\frac{H_{\Delta}^{\prime}(y)}{H_{\Delta}(y)} h-\frac{G_{\Delta}^{\prime}(y)}{G_{\Delta}(y)} h+o(h)\right)\right)^{-1} \\
& \rightarrow 1-G_{\Delta_{1}}(x)-H_{\Delta_{1}}(x) \\
& \left.+H_{\Delta_{1}}(x)\left(-\frac{W_{\Delta}(y)}{H_{\Delta}(y)}+\frac{H_{\Delta}^{\prime}(y)}{H_{\Delta}(y)}\right)\left(\frac{H_{\Delta}^{\prime}(y)}{H_{\Delta}(y)}-\frac{G_{\Delta}^{\prime}(y)}{G_{\Delta}(y)}\right)\right)^{-1} \\
& =1-G_{\Delta_{1}}(x)-H_{\Delta_{1}}(x) \frac{W_{\Delta}(y)+G_{\Delta}(y)\left(W_{\Delta}(y)-H_{\Delta}^{\prime}(y)\right)}{W_{\Delta}(y)} \\
& =1-G_{\Delta_{1}}(x)-H_{\Delta_{1}}(x) \frac{-H_{\Delta}(y) G_{\Delta}^{\prime}(y)+G_{\Delta}(y) W_{\Delta}(y)}{W_{\Delta}(y)} .
\end{aligned}
$$

Раскрывая вероятности первого выхода по формулам

$$
H_{\Delta_{1}}(x)=\frac{H_{\Delta}(x)}{H_{\Delta}(y)}, \quad G_{\Delta_{1}}(x)=G_{\Delta}(x)-G_{\Delta}(y) \frac{H_{\Delta}(x)}{H_{\Delta}(y)},
$$

получаем

$$
\lim _{h \rightarrow 0} P_{x}\left(\Psi_{1}\right)=1-G_{\Delta}(x)+H_{\Delta}(x) \frac{G_{\Delta}^{\prime}(y)}{W_{\Delta}(y)} .
$$


2) Рассмотрим процесс, начинающийся из точки $x \in \Delta_{2}$. Пусть

$$
\begin{aligned}
& \tau_{1}=\sigma_{\Delta_{2}}, \quad \tau_{2}=\tau_{1} \dot{+} \sigma_{\Delta_{1}}, \quad \tau_{3}=\tau_{2} \dot{+} \sigma_{\Delta_{2}}, \quad \tau_{4}=\tau_{3} \dot{+} \sigma_{\Delta_{1}}, \quad \ldots ; \\
& B_{0}=\kappa_{1}\left(\Delta_{2}\right), \quad B_{1}=\theta_{\tau_{1}}^{-1} \kappa_{2}\left(\Delta_{1}\right), \\
& B_{2}=\theta_{\tau_{2}}^{-1} \kappa_{1}\left(\Delta_{2}\right), \quad B_{3}=\theta_{\tau_{3}}^{-1} \kappa_{2}\left(\Delta_{1}\right), \quad \ldots \text {; } \\
& \Psi_{3}=\kappa\left(\Delta_{2}\right)+\sum_{n=1}^{\infty} B_{0} B_{1} \cdots B_{2 n-1} \theta_{\tau_{2 n}}^{-1} \kappa\left(\Delta_{2}\right) \text {; } \\
& \Psi_{4}=\sum_{n=0}^{\infty} B_{0} B_{1} \cdots B_{2 n} \theta_{\tau_{2 n+1}}^{-1} \kappa\left(\Delta_{1}\right) .
\end{aligned}
$$

Имеем $P_{x}\left(\Psi_{3}+\Psi_{4} \mid \sigma_{\Delta}=\infty\right)=1$, кроме того,

$$
\Psi_{3} \subset\left\{\lim _{t \rightarrow \infty} X(t) \geqslant a_{2}\right\}, \quad \Psi_{4} \subset\left\{\lim _{t \rightarrow \infty} X(t) \leqslant b_{1}\right\} .
$$

Отсюда вытекает, что

$$
\begin{aligned}
P_{x}\left(\Psi_{3} \mid \sigma_{\Delta}=\infty\right) & \leqslant P_{x}\left(\lim _{t \rightarrow \infty} X(t) \geqslant a_{2} \mid \sigma_{\Delta}=\infty\right) \\
P_{x}\left(\Psi_{4} \mid \sigma_{\Delta}=\infty\right) & =1-P_{x}\left(\Psi_{3} \mid \sigma_{\Delta}=\infty\right) \leqslant P_{x}\left(\lim _{t \rightarrow \infty} X(t) \leqslant b_{1} \mid \sigma_{\Delta}=\infty\right) \\
& =1-P_{x}\left(\lim _{t \rightarrow \infty} X(t)>b_{1} \mid \sigma_{\Delta}=\infty\right) .
\end{aligned}
$$

Следовательно,

$$
\begin{aligned}
P_{x}\left(\lim _{t \rightarrow \infty} X(t)>b_{1} \mid \sigma_{\Delta}=\infty\right) & \leqslant P_{x}\left(\Psi_{3} \mid \sigma_{\Delta}=\infty\right) \\
& \leqslant P_{x}\left(\lim _{t \rightarrow \infty} X(t) \geqslant a_{2} \mid \sigma_{\Delta}=\infty\right) .
\end{aligned}
$$

При $b_{1} \rightarrow a_{2}$ левая часть этого двойного неравенства стремится к правой в любой точке непрерывности искомой функции распределения. Поэтому

$$
P_{x}\left(\lim _{t \rightarrow \infty} X(t) \geqslant a_{2} \mid \sigma_{\Delta}=\infty\right)=\lim _{b_{1} \rightarrow a_{2}} P_{x}\left(\Psi_{3} \mid \sigma_{\Delta}=\infty\right) .
$$

Пусть $a_{2}=y$ и $b_{1}=y+h$. Имеем

$$
\begin{aligned}
P_{x}\left(\Psi_{3}\right)= & 1-G_{\Delta_{2}}(x)-H_{\Delta_{2}}(x) \\
& +\sum_{n=0}^{\infty} G_{\Delta_{2}}(x) H_{\Delta_{1}}(y)\left(G_{\Delta_{2}}(y+h) H_{\Delta_{1}}(y)\right)^{n} \\
& \times\left(1-G_{\Delta_{2}}(y+h)-H_{\Delta_{2}}(y+h)\right) \\
= & 1-G_{\Delta_{2}}(x)-H_{\Delta_{2}}(x) \\
& +G_{\Delta_{2}}(x) H_{\Delta_{1}}(y) \frac{1-G_{\Delta_{2}}(y+h)-H_{\Delta_{2}}(y+h)}{1-G_{\Delta_{2}}(y+h) H_{\Delta_{1}}(y)} .
\end{aligned}
$$


Пользуясь дифференцируемостью переходных функций, получаем

$$
P_{x}\left(\Psi_{3}\right) \rightarrow 1-H_{\Delta}(x)-G_{\Delta}(x) \frac{H_{\Delta}^{\prime}(y)}{W_{\Delta}(y)} \quad(h \rightarrow 0),
$$

откуда следует, что

$$
\begin{aligned}
Q_{\Delta}(y \mid x) & =1-\left(1-H_{\Delta}(x)-G_{\Delta}(x)\right)^{-1}\left(1-H_{\Delta}(x)-G_{\Delta}(x) \frac{H_{\Delta}^{\prime}(y)}{W_{\Delta}(y)}\right) \\
& =\left(1-H_{\Delta}(x)-G_{\Delta}(x)\right)^{-1}\left(-G_{\Delta}(x)+G_{\Delta}(x) \frac{H_{\Delta}^{\prime}(y)}{W_{\Delta}(y)}\right) .
\end{aligned}
$$

Теорема 2 доказана.

Следствие 1. Из условия на коэффициенты уравнения (7) следует, что функиия распределения $Q_{\Delta}$ имеет плотность следующего вида:

1) $п р и ~ y>x$

$$
Q_{\Delta}^{\prime}(y \mid x)=\frac{2 B(y) G_{\Delta}(y) H_{\Delta}(x)}{W_{\Delta}(y)\left(1-G_{\Delta}(x)-H_{\Delta}(x)\right)} ;
$$

2) при $y<x$

$$
Q_{\Delta}^{\prime}(y \mid x)=\frac{2 B(y) H_{\Delta}(y) G_{\Delta}(x)}{W_{\Delta}(y)\left(1-G_{\Delta}(x)-H_{\Delta}(x)\right)} .
$$

Д о к а з а т е л ь с т в о. Рассмотрим производную вронскиана. Пользуясь тем, что функции $G_{\Delta}$ и $H_{\Delta}$ являются решениями уравнения (7), получаем

$$
\begin{aligned}
W_{\Delta}^{\prime} & =\left(H_{\Delta}^{\prime} G_{\Delta}-H_{\Delta} G_{\Delta}^{\prime}\right)^{\prime}=H_{\Delta}^{\prime \prime} G_{\Delta}-H_{\Delta} G_{\Delta}^{\prime \prime} \\
& =\left(-2 A(y) H_{\Delta}^{\prime}+2 B(y) H_{\Delta}\right) G_{\Delta}-\left(-2 A(y) G_{\Delta}^{\prime}+2 B(y) G_{\Delta}\right) H_{\Delta} \\
& =-2 A(y)\left(H_{\Delta}^{\prime} G_{\Delta}-H_{\Delta} G_{\Delta}^{\prime}\right)=-2 A(y) W_{\Delta},
\end{aligned}
$$

откуда, в частности, следует, что

$$
W_{\Delta}(y)=W_{\Delta}(a) \exp \left(-\int_{a}^{y} 2 A(s) d s\right) .
$$

1) При $y>x$ имеем

$$
\begin{aligned}
\left(\frac{G_{\Delta}^{\prime}(y)}{W_{\Delta}(y)}\right)^{\prime} & =\frac{G_{\Delta}^{\prime \prime}(y) W_{\Delta}(y)-W_{\Delta}^{\prime}(y) G_{\Delta}^{\prime}(y)}{W_{\Delta}^{2}(y)} \\
& =\frac{\left(-2 A(y) G_{\Delta}^{\prime}(y)+2 B(y) G_{\Delta}(y)\right) W_{\Delta}(y)+2 A(y) W_{\Delta}(y) G_{\Delta}^{\prime}(y)}{W_{\Delta}^{2}(y)} \\
& =\frac{2 B(y) G_{\Delta}(y) W_{\Delta}(y)}{W_{\Delta}^{2}(y)}=\frac{2 B(y) G_{\Delta}(y)}{W_{\Delta}(y)} .
\end{aligned}
$$


2) Аналогично, при $y<x$ имеем

$$
\left(\frac{H_{\Delta}^{\prime}(y)}{W_{\Delta}(y)}\right)^{\prime}=\frac{2 B(y) H_{\Delta}(y)}{W_{\Delta}(y)}
$$

Следствие доказано.

П р и м е р. Рассмотрим уравнение (7) на интервале $\Delta=(a, b)$ с коэффициентами $A(x) \equiv A, B(0, x) \equiv B$. Стандартным способом находим два решения задачи Дирихле:

$$
G_{\Delta}(x)=e^{-A(x-a)} \frac{\operatorname{sh}(b-x) r}{\operatorname{sh}(b-a) r}, \quad H_{\Delta}(x)=e^{A(b-x)} \frac{\operatorname{sh}(x-a) r}{\operatorname{sh}(b-a) r},
$$

где $r=\sqrt{A^{2}+2 B}($ см. [1]). При этом

$G_{\Delta}^{\prime}=-A G_{\Delta}-r e^{-A(x-a)} \frac{\operatorname{ch}(b-x) r}{\operatorname{sh}(b-a) r}, \quad H_{\Delta}^{\prime}=-A H_{\Delta}+r e^{A(b-x)} \frac{\operatorname{ch}(x-a) r}{\operatorname{sh}(b-a) r}$,

откуда следует, что

$$
\begin{aligned}
W_{\Delta}= & \left(-A H_{\Delta}+r e^{A(b-x)} \frac{\operatorname{ch}(x-a) r}{\operatorname{sh}(b-a) r}\right) G_{\Delta} \\
& -\left(-A G_{\Delta}-r e^{-A(x-a)} \frac{\operatorname{ch}(b-x) r}{\operatorname{sh}(b-a) r}\right) H_{\Delta} \\
= & \frac{r}{\operatorname{sh}^{2}(b-a) r} e^{A(b-2 x+a)} \\
& \times[\operatorname{sh}(b-x) r \operatorname{ch}(x-a) r+\operatorname{sh}(x-a) r \operatorname{ch}(b-x) r] \\
= & \frac{r}{\operatorname{sh}(b-a) r} e^{A(b-2 x+a)} .
\end{aligned}
$$

1) При $y>x, a \rightarrow-\infty, b \rightarrow \infty$ имеем

$$
\begin{aligned}
\frac{2 B G_{\Delta}(y) H_{\Delta}(x)}{W_{\Delta}(y)} & \\
= & \frac{2 B}{r} \frac{\exp (a-y) A \frac{\operatorname{sh}(b-y) r}{\operatorname{sh}(b-a) r} \exp (b-x) A \frac{\operatorname{sh}(x-a) r}{\operatorname{sh}(b-a) r}}{\exp ((b-2 y+a) A) / \operatorname{sh}(b-a) r} \\
& \times \frac{\exp (b-x) A \operatorname{sh}(x-a) r}{\operatorname{sh}(b-a) r} \\
= & \frac{2 B}{r} \exp (y-x) A \frac{\operatorname{sh}(b-y) r \operatorname{sh}(x-a) r}{\operatorname{sh}(b-a) r} \\
\sim & \frac{B}{r} \exp (y-x) A \frac{\exp (b-y) r \exp (x-a) r}{\exp (b-a) r}=\frac{B}{r} e^{-(r-A)(y-x)} .
\end{aligned}
$$

2) При $y<x, a \rightarrow-\infty, b \rightarrow \infty$ имеем

$$
\frac{2 B H_{\Delta}(y) G_{\Delta}(x)}{W_{\Delta}(y)}=\frac{2 B}{r} \frac{\exp (b-y) A \operatorname{sh}(y-a) r / \operatorname{sh}(b-a) r}{\exp (b-2 y+a) A / \operatorname{sh}(b-a) r}
$$




$$
\begin{aligned}
& \times \frac{\exp (a-x) A \operatorname{sh}(b-x) r}{\operatorname{sh}(b-a) r} \\
= & \frac{2 B}{r} \exp (y-x) A \frac{\operatorname{sh}(y-a) r \operatorname{sh}(b-x) r}{\operatorname{sh}(b-a) r} \\
\sim & \frac{B}{r} \exp (y-x) A \frac{\exp (y-a) r \exp (b-x) r}{\exp (b-a) r} \\
= & \frac{B}{r} e^{-(r+A)(x-y)} .
\end{aligned}
$$

Замечая, что при $a \rightarrow-\infty, b \rightarrow \infty$ имеет место сходимость

$$
1-G_{\Delta}(x)-H_{\Delta}(x) \rightarrow 1
$$

получаем

$$
Q_{(a, b)}^{\prime}(y \mid x) \rightarrow \begin{cases}\frac{B}{r} e^{-(r-A)(y-x)}, & \text { если } y>x, \\ \frac{B}{r} e^{-(r+A)(x-y)}, & \text { если } y<x .\end{cases}
$$

\section{СПИСОК ЛИТЕРАТУРЫ}

1. Бородин А.Н., Салминен П. Справочник по броуновскому движению. СПб.,: Лань, 2000, 639 с.

2. Дынкин Е. Б. Марковские процессы. М: Физматлит, 1963, 859 с.

3. Ито К., Маккин Г. Диффузионные процессы и их траектории. М.: Мир, 1968, 394 c.

4. Камке Э. Справочник по обыкновенным дифференциальным уравнениям. М.: Наука, 1971, 576 с.

5. Королюк В. С., Турбин А. Ф. Полумарковские процессы и их приложения. Киев: Наукова думка, 1976, $184 \mathrm{c.}$

6. Харламов Б. П. Непрерывные полумарковские процессы. СПб.: Наука, 2001, $431 \mathrm{c.}$

7. Харламов Б. П. Диффузионный процесс с задержкой на границах отрезка. - Зап. науч. сем. ПОМИ, 2007, т. 351, с. 284-297.

8. Харламов Б. П. О марковском диффузионном процессе с замедленным отражением на границе интервала. - Зап. науч. сем. ПОМИ, 2009, т. 368, с. 243-267.

9. Харламов Б. П. О точках задержки и асимметрии одномерного полумарковского диффузионного процесса. - Зап. науч. сем. ПОМИ, 2010, т. 384, с. 291-309.

10. Расова С. С., Харламов Б. П. О движении броуновских частиц вдоль задерживающего экрана. - Зап. науч. сем. ПОМИ, 2011, т. 396, с. 175-194.

11. Harlamov B. P. Continuous Semi-Markov Processes. London/Hoboken: ISTE/Wiley, 2008, 375 p.

12. Harlamov B. P. Stochastic model of gas capillary chromatography. - Comm. Statist. Simulation Comput., 2012, v. 41, № 7, p. 1023-1031.

Поступила в редакцию

28.IV.2014

Исправленный вариант

13.II. 2015 